\title{
Status of neutrino oscillations II: How to reconcile the LSND result?
}

\section{Thomas Schwetz*}

Institut für Theoretische Physik, Physik Department

Technische Universität München, James-Franck-Str., D-85748 Garching, Germany

E-mail: ischwetz@ph.tum.de'

ABstract: We present an analysis of the global neutrino oscillation data including the LSND result in terms of four-neutrino mass schemes and a CPT violating three-neutrino framework. We find that the strong preference of oscillations into active neutrinos implied by recent solar+KamLAND as well as atmospheric neutrino data allows to rule out $(2+2)$ mass schemes at $5.8 \sigma$, whereas $(3+1)$ schemes are disfavoured at the $3.2 \sigma$ level by short-baseline experiments. The CPT violating scenario is disfavoured at $3.4 \sigma$ by KamLAND and atmospheric anti-neutrino data. We conclude that currently no convincing explanation for the LSND signal exists, and it remains a puzzle how to reconcile it with the other evidences for neutrino oscillations.

\section{Introduction}

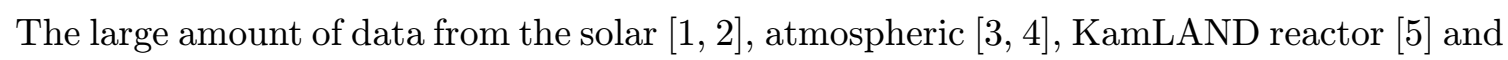
$\mathrm{K} 2 \mathrm{~K}$ accelerator [6] neutrino experiments is beautifully described in terms of three-flavour neutrino oscillations $[\bar{i} \bar{i}]$. To reconcile also the evidence for oscillations from the LSND experiment [i. To obtain the required three neutrino mass-squared differences of different orders of magnitude it has been proposed to introduce a light sterile neutrino [igi, or to allow for violation

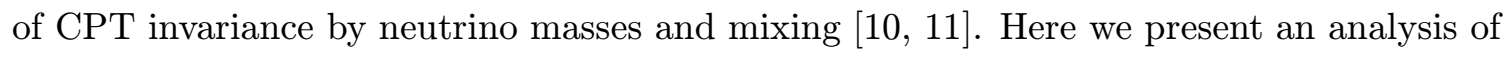
the global neutrino oscillation data in terms of four-neutrino and CPT violating schemes, including data from solar, atmospheric, KamLAND and K2K neutrino experiments, the LSND experiment, as well as data from short-baseline (SBL) experiments [1]2, reactor experiments [ī1]

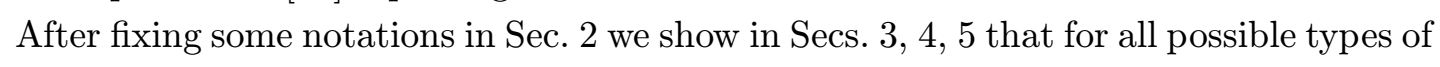
four-neutrino schemes different sub-sets of the data are in serious disagreement and hence,

${ }^{*}$ Speaker. 

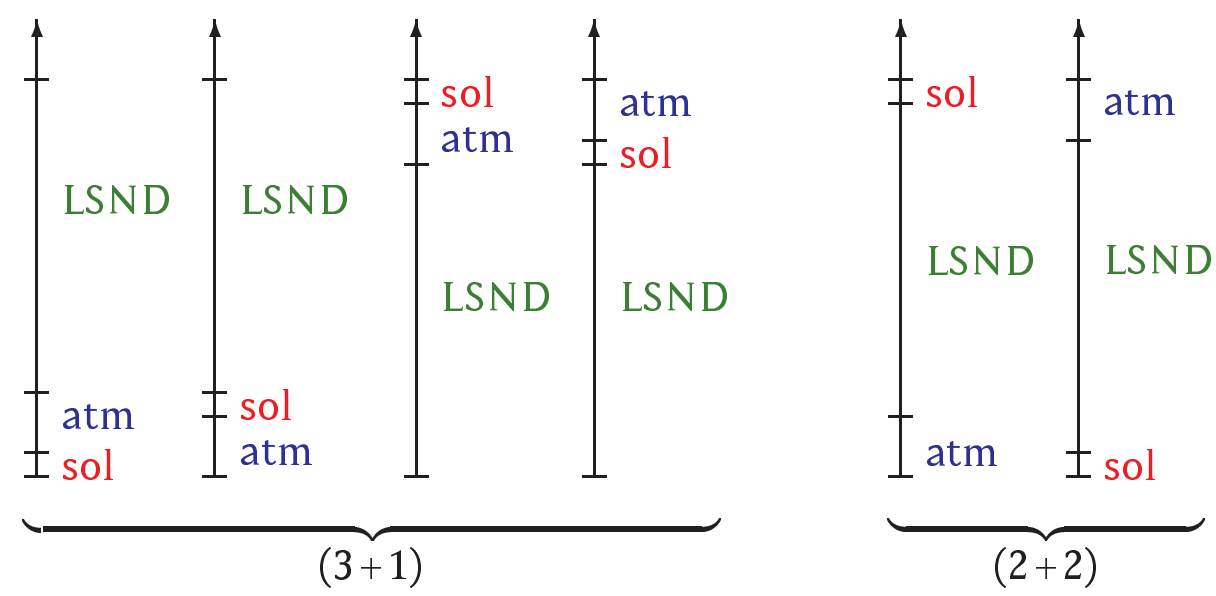

Figure 1: The six four-neutrino mass spectra, divided into the classes $(3+1)$ and $(2+2)$.

four-neutrino oscillations do not provide a satisfactory description of the global oscillation

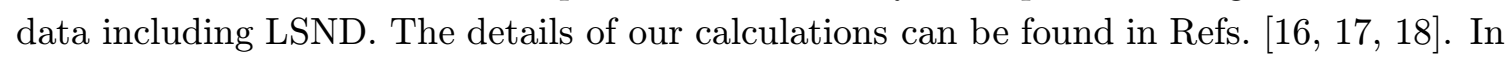

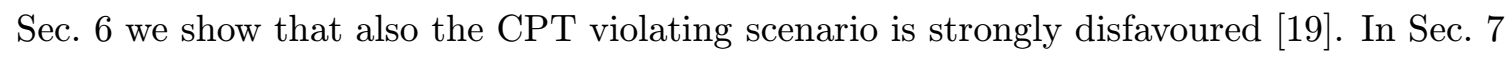
we briefly comment on some proposed alternative solutions for the LSND puzzle, and we summarize our results in Sec. ${ }_{-i}^{8}$

\section{Four-neutrino schemes: notations and approximations}

Four-neutrino mass schemes are usually divided into the two classes $(3+1)$ and $(2+2)$, as illustrated in Fig. $i_{-i}^{i}$ We note that $(3+1)$ mass spectra include the three-active neutrino scenario as limiting case. In this case solar and atmospheric neutrino oscillations are explained by active neutrino oscillations, with mass-squared differences $\Delta m_{\mathrm{SOL}}^{2}$ and $\Delta m_{\mathrm{ATM}}^{2}$, and the fourth neutrino state gets completely decoupled. We will refer to such limiting scenario as $(3+0)$. In contrast, the $(2+2)$ spectrum is intrinsically different, as there must be a significant contribution of the sterile neutrino either in solar or in atmospheric neutrino oscillations or in both.

Neglecting CP violation, in general neutrino oscillations in four-neutrino schemes are described by 9 parameters: 3 mass-squared differences and 6 mixing angles in the unitary lepton mixing matrix. Here we use a parameterisation introduced in Ref. [i $\left.\mathbf{i}_{i}^{2}\right]$, which is based on physically relevant quantities: the 6 parameters $\Delta m_{\mathrm{SOL}}^{2}, \theta_{\mathrm{SOL}}, \Delta m_{\mathrm{ATM}}^{2}, \theta_{\mathrm{ATM}}$, $\Delta m_{\mathrm{LSND}}^{2}, \theta_{\mathrm{LSND}}$ are similar to the two-neutrino mass-squared differences and mixing angles and are directly related to the oscillations in solar, atmospheric and the LSND experiments. For the remaining 3 parameters we use $\eta_{s}, \eta_{e}$ and $d_{\mu}$. Here, $\eta_{s}\left(\eta_{e}\right)$ is the fraction of $\nu_{s}\left(\nu_{e}\right)$ participating in solar oscillations, and $\left(1-d_{\mu}\right)$ is the fraction of $\nu_{\mu}$ participating in oscillations with $\Delta m_{\text {AтM }}^{2}$ (for exact definitions see Ref. [1] $\left.\underline{1}_{i} \bar{\theta}_{1}\right)$. For the analysis we adopt the following approximations:

1. We make use of the hierarchy $\Delta m_{\mathrm{SOL}}^{2} \ll \Delta m_{\mathrm{ATM}}^{2} \ll \Delta m_{\mathrm{LSND}}^{2}$. This means that for each data set we consider only one mass-squared difference, the other two are set either to zero or to infinity. 


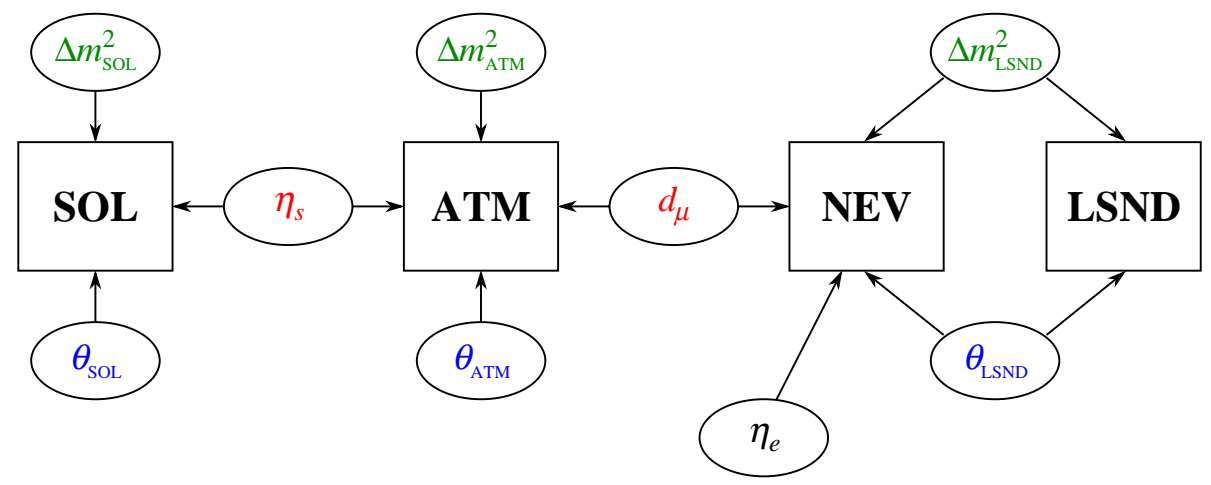

Figure 2: Parameter dependence of the different data sets in our parameterisation.

2. In the analyses of solar and atmospheric data (but not for SBL data) we set $\eta_{e}=1$,

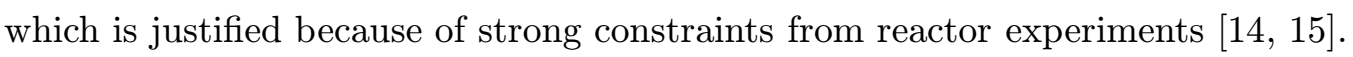

Due to these approximations the parameter structure of the four-neutrino analysis gets rather simple. The parameter dependence of the four data sets solar, atmospheric, LSND and NEV is illustrated in Fig.

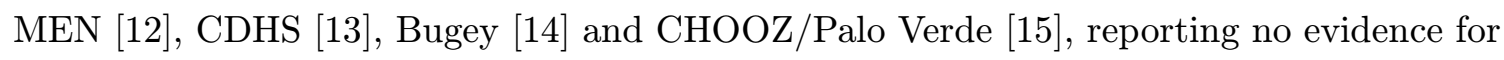
oscillations. We see that only $\eta_{s}$ links solar and atmospheric data and $d_{\mu}$ links atmospheric and NEV data. LSND and NEV data are coupled by $\Delta m_{\mathrm{LSND}}^{2}$ and $\theta_{\mathrm{LSND}}$.

\section{3. $(2+2)$ : ruled out by solar and atmospheric data}

The strong preference of oscillations into active neutrinos in solar and atmospheric oscillations leads to a direct conflict in $(2+2)$ oscillation schemes. We will now show that thanks to recent solar neutrino data (in particular from SNO [2in] ) in combination with the KamLAND experiment [5] and the latest SK data on atmospheric neutrinos [3i] the tension in the data has become so strong that $(2+2)$ oscillation schemes are essentially ruled out. ${ }^{1}$

In the left panel of Fig. $\underline{3}_{1}$ we show the $\Delta \chi^{2}$ from solar neutrino data as a function of $\eta_{s}$, the parameter describing the fraction of the sterile neutrino participating in solar neutrino oscillations. One can see that the improved determination of the neutral current event rate from the solar ${ }^{8} \mathrm{~B}$ flux implied by the salt enhanced measurement in SNO [2] leads to a significant tightening of the constraint on $\eta_{s}$ : the 99\% CL bound improves from $\eta_{s} \leq 0.44$ for pre-SNO salt to $\eta_{s} \leq 0.31$ including also the most recent SNO data. Moreover, large values of $\eta_{s} \gtrsim 0.5$ are now ruled out by many standard deviations. The outstanding results of the KamLAND reactor experiment confirmed the LMA solution of the solar neutrino problem [20 $\left.\overline{2}_{1}^{1}, \overline{2} \overline{2} \overline{2}\right]$. Apart from this very important result the impact of KamLAND for an admixture of a sterile neutrino is rather limited (see Fig. 统). Since KamLAND on its own has no sensitivity to $\eta_{s}$ the bound is only indirectly affected due to the better determination of $\Delta m_{\mathrm{SOL}}^{2}$ and $\theta_{\mathrm{SOL}}$. The combined analysis leads to the $99 \%$ CL bound $\eta_{s} \leq 0.27$.

\footnotetext{
${ }^{1}$ Details of our analyses of the solar, KamLAND and atmospheric neutrino data can be found in Refs. 'ilí, [201]. For an earlier four-neutrino analysis of solar and atmospheric data see Ref. [1]
} 

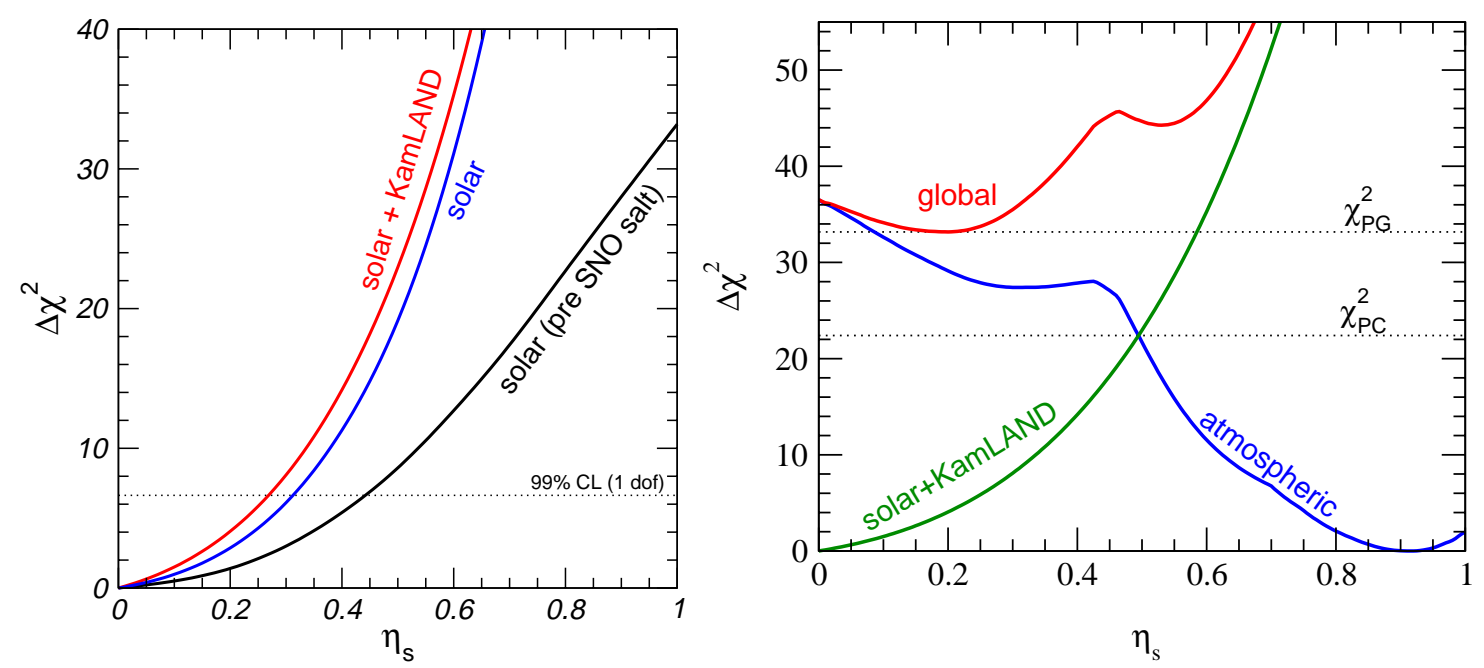

Figure 3: Left: $\Delta \chi^{2}$ from solar and KamLAND data as a function of $\eta_{s}$. Right: $\Delta \chi_{\mathrm{SOL}}^{2}, \Delta \chi_{\mathrm{ATM}+\mathrm{SBL}}^{2}$ and $\bar{\chi}_{\text {global }}^{2}$ as a function of $\eta_{s}$ in $(2+2)$ oscillation schemes.

In contrast, in $(2+2)$ schemes atmospheric data imply $\eta_{s} \geq 0.65$ at $99 \% \mathrm{CL}$, in clear disagreement with the bound from solar data. In the right panel of Fig. ${ }_{-1}^{3} \overline{1}$ we show the $\Delta \chi^{2}$ for solar data and for atmospheric combined with SBL data as a function of $\eta_{s}$. Furthermore, we show the $\chi^{2}$ of the global data defined by

$$
\bar{\chi}^{2}\left(\eta_{s}\right) \equiv \Delta \chi_{\mathrm{SOL}}^{2}\left(\eta_{s}\right)+\Delta \chi_{\mathrm{ATM}+\mathrm{SBL}}^{2}\left(\eta_{s}\right) .
$$

From the figure we find that only at the $4.7 \sigma$ level a value of $\eta_{s}$ exists, which is contained in the allowed regions of both data sets. This follows from the $\chi^{2}$-value $\chi_{\mathrm{PC}}^{2}=22.4$ shown in the figure. In Refs. [1] $\left.\overline{1}_{-1}^{1}, \overline{2} \overline{2} \overline{3}\right]$ we have proposed a statistical method to evaluate the disagreement of different data sets in global analyses. The parameter goodness of fit (PG) makes use of the $\bar{\chi}^{2}$ defined in Eq. $\left(\overline{3} . \overline{1}_{1}\right)$. This criterion evaluates the GOF of the combination of data sets, without being diluted by a large number of data points, as it

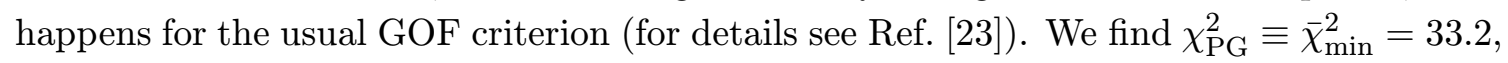
which corresponds to $5.7 \sigma$. We conclude that $(2+2)$ mass schemes are ruled out by the disagreement between the latest solar and atmospheric neutrino data. This is a very robust result, independent of whether LSND is confirmed or disproved. ${ }^{2}$

\section{4. $(3+1)$ : strongly disfavoured by SBL data}

It is known for a long time [20 2 in] that $(3+1)$ mass schemes are disfavoured by the comparison

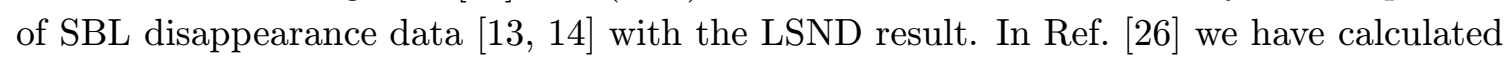

\footnotetext{
${ }^{2}$ Sub-leading effects beyond the approximations adopted here should not affect this result significantly. Allowing for additional parameters to vary at the percent level might change the ratio of some observables [24], however, we expect that the absolute number of events relevant for the fit will not change substantially.
} 


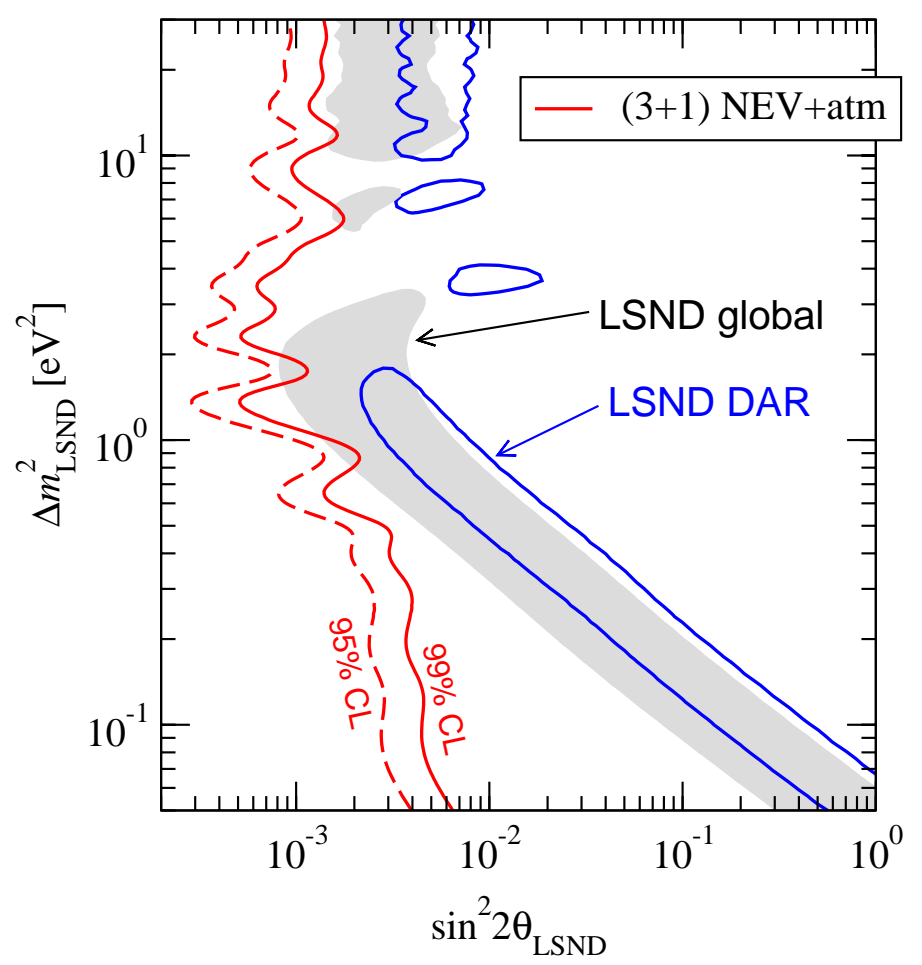

Figure 4: Upper bound on $\sin ^{2} 2 \theta_{\mathrm{LSND}}$ from NEV and atmospheric neutrino data in $(3+1)$

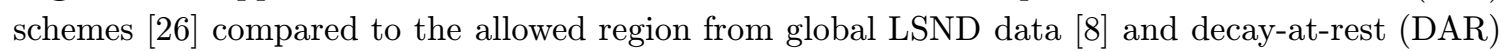
LSND data [2글.

an upper bound on the LSND oscillation amplitude $\sin ^{2} 2 \theta_{\text {LSND }}$ resulting from NEV and atmospheric neutrino data. From Fig. 'is-is we see that this bound is incompatible with the signal observed in LSND at the 95\% CL. Only marginal overlap regions exist between the bound and global LSND data if both are taken at 99\% CL. An analysis in terms of the parameter goodness of fit [i $\left[\bar{z}_{1}\right]$ shows that for most values of $\Delta m_{\mathrm{LSND}}^{2} \mathrm{NEV}$ and atmospheric data are compatible with LSND only at more than $3 \sigma$, with one exception around $\Delta m_{\mathrm{LSND}}^{2} \sim 6 \mathrm{eV}^{2}$, where the $\mathrm{PG}$ reaches $1 \%$. These results show that $(3+1)$ schemes are strongly disfavoured by SBL disappearance data.

\section{Comparing $(3+1),(2+2)$ and $(3+0)$ hypotheses}

With the methods developed in Ref. [1] $]_{1}^{1}$ ] we are able to perform a global fit to the oscillation data in the four-neutrino framework. This approach allows to statistically compare the different hypotheses. Let us first evaluate the GOF of $(3+1)$ and $(2+2)$ spectra with the help of the PG method described in Ref. [2.23]. We divide the global oscillation data in the 4 data sets SOL, ATM, LSND and NEV. Following Ref. [i] $\left.\overline{1}_{2}\right]$ we consider

$$
\begin{aligned}
\bar{\chi}^{2} & =\Delta \chi_{\mathrm{SOL}}^{2}\left(\theta_{\mathrm{SOL}}, \Delta m_{\mathrm{SOL}}^{2}, \eta_{s}\right)+\Delta \chi_{\mathrm{ATM}}^{2}\left(\theta_{\mathrm{ATM}}, \Delta m_{\mathrm{ATM}}^{2}, \eta_{s}, d_{\mu}\right) \\
& +\Delta \chi_{\mathrm{NEV}}^{2}\left(\theta_{\mathrm{LSND}}, \Delta m_{\mathrm{LSND}}^{2}, d_{\mu}, \eta_{e}\right)+\Delta \chi_{\mathrm{LSND}}^{2}\left(\theta_{\mathrm{LSND}}, \Delta m_{\mathrm{LSND}}^{2}\right),
\end{aligned}
$$




\begin{tabular}{|c|cccc|c|c|}
\hline & SOL & ATM & LSND & NEV & $\chi_{\mathrm{PG}}^{2}$ & PG \\
\hline$(3+1)$ & 0.0 & 0.4 & 7.2 & 7.0 & 14.6 & $5.6 \times 10^{-3}(3.2 \sigma)$ \\
$(2+2)$ & 4.1 & 29.2 & 1.2 & 9.7 & 44.1 & $6.1 \times 10^{-9}(5.8 \sigma)$ \\
\hline
\end{tabular}

Table 1: Parameter GOF and the contributions of different data sets to $\chi_{\mathrm{PG}}^{2}$ in $(3+1)$ and $(2+2)$ neutrino mass schemes.

where $\Delta \chi_{X}^{2}=\chi_{X}^{2}-\left(\chi_{X}^{2}\right)_{\min }(X=\mathrm{SOL}, \mathrm{ATM}, \mathrm{NEV}, \mathrm{LSND})$. In Tab. 嶭 we show the contributions of the 4 data sets to $\chi_{\mathrm{PG}}^{2} \equiv \bar{\chi}_{\text {min }}^{2}$ for $(3+1)$ and $(2+2)$ oscillation schemes. As expected we observe that in $(3+1)$ schemes the main contribution comes from SBL data due to the tension between LSND and NEV data in these schemes. For $(2+2)$ oscillation schemes a large part of $\chi_{\mathrm{PG}}^{2}$ comes from solar and atmospheric data, however, also SBL data contributes significantly. This comes mainly from the tension between LSND and KARMEN [2] $\overline{2}]$, which does not depend on the mass scheme and, hence, also contributes in the case of $(2+2)$. Therefore, the values of $\chi_{\mathrm{PG}}^{2}$ in Tab. 点 for $(2+2)$ schemes are higher than the one given in Sec. $\overline{3}_{-1}$, where the tension in SBL data is not included.

The parameter goodness of fit is now obtained by evaluating $\chi_{\mathrm{PG}}^{2}$ for 4 DOF [ $[\overline{2} \overline{3} \overline{-}]$. This number of degrees of freedom corresponds to the 4 parameters $\eta_{s}, d_{\mu}, \theta_{\mathrm{LSND}}, \Delta m_{\mathrm{LSND}}^{2}$ describing the coupling of the different data sets (see Eq. ( $(\overline{2}, \overline{1} \cdot \overline{1})$ and Fig. is obtained in the $(3+1)$ case. However, even in this best case the PG is only $0.56 \%$. The $\mathrm{PG}$ of $6.1 \times 10^{-9}$ for $(2+2)$ schemes shows that these mass schemes are essentially ruled out by the disagreement between the individual data sets.

Although we have seen that none of the four-neutrino mass schemes can provide a reasonable good fit to the global oscillation data including LSND, it might be interesting to consider the relative status of the three hypotheses $(3+1),(2+2)$ and the three-active neutrino scenario $(3+0)$. This can be done by comparing the $\chi^{2}$ values of the best fit point - which occurs for the $(3+1)$ scheme - with the one corresponding to $(2+2)$ and $(3+0)$. First we observe that $(2+2)$ schemes are strongly disfavoured with respect to $(3+1)$ with a $\Delta \chi^{2}=29.5$. For $4 \mathrm{DOF}$ this is equivalent to an exculsion at $4.5 \sigma$. Furthermore, we find that $(3+0)$ is disfavoured with a $\Delta \chi^{2}=20.0(99.95 \%$ CL for 4 DOF) with respect to $(3+1)$. This reflects the high statistical significance of the LSND result, since in a $(3+0)$ no effect is predicted for LSND.

\section{CPT violation}

The CPT violating solution for the LSND results proposed in Refs. [1] $\left.{ }_{1}^{1}\right]$ is based on the observation that the signal in favour of oscillations in LSND is dominated by the antineutrino signal from decay-at-rest data. The neutrino data from the decay-in-flight event sample gives a $\nu_{\mu} \rightarrow \nu_{e}$ transition probability of $(0.10 \pm 0.16 \pm 0.04) \%$ [ [i]i] consistent with the anti-neutrino signal of $P_{\bar{\nu}_{\mu} \rightarrow \bar{\nu}_{e}}=(0.264 \pm 0.067 \pm 0.045) \%$, is also perfectly consistent with the absence of neutrino oscillations in LSND. Therefore, it has been proposed to adopt different masses and mixings for neutrinos and anti-neutrinos

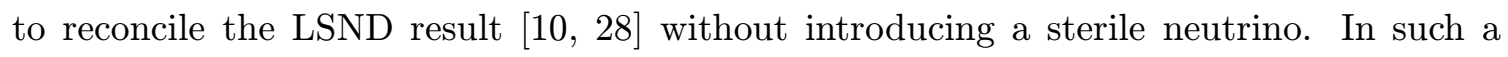


$\overline{\mathrm{m}}_{3}$

Atmospheric, LSND

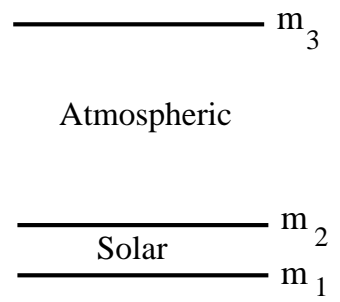

Neutrinos

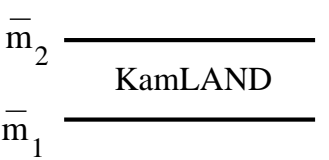

Antineutrinos

Figure 5: CPT violating neutrino mass scheme proposed in Ref. [1]

scheme solar and atmospheric neutrino oscillations are driven by $\Delta m_{\mathrm{SOL}}^{2}$ and $\Delta m_{\mathrm{ATM}}^{2}$, and atmospheric and LSND anti-neutrino oscillations by $\Delta \bar{m}_{\mathrm{ATM}}^{2}$ and $\Delta \bar{m}_{\mathrm{LSND}}^{2}$.

This scenario is strongly affected by the KamLAND results on anti-neutrino disappearance, which in combination with the constraints from CHOOZ indicate oscillations with $\Delta \bar{m}^{2} \lesssim 10^{-4} \mathrm{eV}^{2}$. Since this requires a third mass scale in the anti-neutrino sector the CPT violating solution seems to be disfavoured. However, in Ref. [1] in in was claimed that the mass spectrum shown in Fig. 雨still may provide a fit to the data, although no anti-neutrino mass-squared difference in the range required for atmospheric oscillations is present. This claim is based on the fact that Super-Kamiokande cannot distinguish between neutrinos and anti-neutrinos, and the atmospheric data sample is dominated by neutrinos by a factor $2-4$ over anti-neutrinos.

In Ref. [i] $1 \overline{9}$ i] we have performed an anlysis of the global oscillation data in the framework of the CPT violating neutrino scheme. First we have analized all data except LSND from solar, atmospheric, reactor (KamLAND, CHOOZ, Bugey) and K2K experiments in a general three-flavour context, by allowing for different neutrino and anti-neutrino oscillation parameters. We found that there is no evidence for any CPT violation in that data set. In fact, the best fit point occurs very close to CPT conservation, and the best fit in the CPT conserving case has $\Delta \chi^{2}$ of only 0.5 .

In Fig. ' ${ }_{-1}^{\prime}$ we show the allowed regions of the all-but-LSND data compared to the LSND allowed regions, projected onto the plane of the LSND oscillation parameters $\Delta m_{\mathrm{LSND}}^{2}=$ $\Delta \bar{m}_{31}^{2}$ and $\sin ^{2} 2 \theta_{\mathrm{LSND}}=\sin ^{2} \bar{\theta}_{23} \sin ^{2} 2 \bar{\theta}_{13}$. This figure illustrates that below $3 \sigma$ CL there is no overlap between the allowed region of the LSND analysis and the all-but-LSND one, and that for this last one the region is restricted to $\Delta m_{\mathrm{LSND}}^{2}<0.02 \mathrm{eV}^{2}$. At higher CL values of $\Delta m_{\mathrm{LSND}}^{2} \sim \mathcal{O}\left(\mathrm{eV}^{2}\right)$ become allowed - as determined mainly by the constraints from Bugey - and an agreement becomes possible. We find that in the neighbourhood of $\Delta m_{\mathrm{LSND}}^{2}=0.9 \mathrm{eV}^{2}$ and $\sin ^{2} 2 \theta_{\mathrm{LSND}}=0.01$ the LSND and the all-but-LSND allowed regions start having some marginal agreement slightly above $3 \sigma$ CL (at $\Delta \chi^{2}=12.2$ ). A 


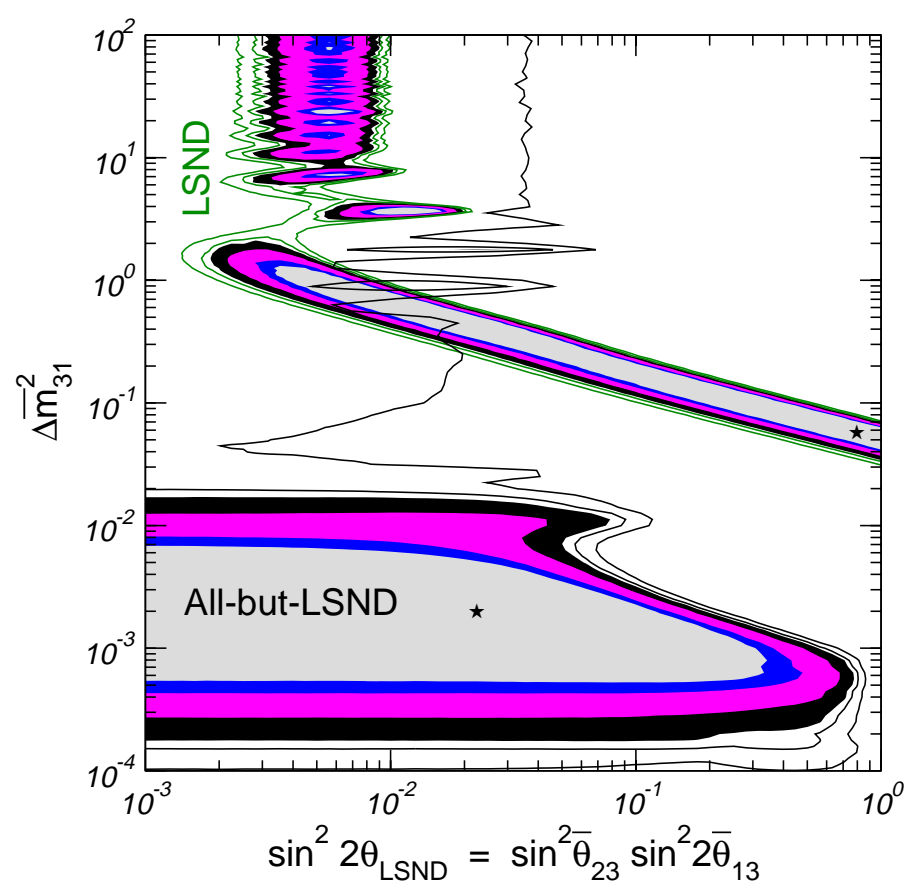

Figure 6: $90 \%, 95 \%, 99 \%$, and $3 \sigma$ CL allowed regions (filled) in the $\left(\Delta m_{\mathrm{LSND}}^{2}, \sin ^{2} 2 \theta_{\mathrm{LSND}}\right)$ plane required to explain the LSND signal together with the corresponding allowed regions from our global analysis of all-but-LSND data. The contour lines correspond to $\Delta \chi^{2}=13$ and $16(3.2 \sigma$ and $3.6 \sigma$, respectively).

less fine-tuned agreement appears at $3.3 \sigma \mathrm{CL}\left(\Delta \chi^{2} \sim 14\right)$ for $\Delta m_{\mathrm{LSND}} \gtrsim 0.5 \mathrm{eV}^{2}$ and $\sin ^{2} 2 \theta_{\mathrm{LSND}} \lesssim 0.01$.

Alternatively the quality of the joint description of LSND and all the other data can be evaluated by performing a global fit based on the total $\chi^{2}$-function $\chi_{\text {tot }}^{2}=\chi_{\text {all-but-LSND }}^{2}+$ $\chi_{\text {LSND }}^{2}$, and applying a goodness-of-fit test. The best fit point of the global analysis is $\sin ^{2} 2 \theta_{\mathrm{LSND}}=6.3 \times 10^{-3}$ and $\Delta m_{\mathrm{LSND}}^{2}=0.89 \mathrm{eV}^{2}$. In the following we will again use the parameter goodness-of-fit [2] $\overline{2} \overline{3}]$, which is particularly suitable to test the compatibility of independent data sets. Applying this method to the present case we consider the statistic

$$
\bar{\chi}^{2}=\Delta \chi_{\text {all-but-LSND }}^{2}(\text { b.f. })+\Delta \chi_{\text {LSND }}^{2} \text { (b.f.) }
$$

where b.f. denotes the global best fit point. The $\bar{\chi}^{2}$ of Eq. $(\overline{6} \cdot \overline{1})$ has to be evaluated for 2 dof, corresponding to the two parameters $\sin ^{2} 2 \theta_{\mathrm{LSND}}$ and $\Delta \bar{m}_{31}^{2}=\Delta m_{\mathrm{LSND}}^{2}$ coupling the two data sets. From $\Delta \chi_{\text {all-but-LSND }}^{2}=12.7$ and $\Delta \chi_{\text {LSND }}^{2}=1.7$ we obtain $\bar{\chi}^{2}=14.4$ leading to the marginal parameter goodness-of-fit of $7.5 \times 10^{-4}$, corresponding to $3.4 \sigma$.

These results show that atmospheric data is sensitive enough to anti-neutrino oscillations, and hence, no reasonable fit can be obtained without a mass-squared difference of the order $10^{-3} \mathrm{eV}^{2}$ for anti-neutrinos. Consequently the CPT violation scenario is disfavoured by global anti-neutrino data from reactor, atmospheric and the LSND experiments. 


\section{Alternative solutions}

In this section we briefly mention some alternative solutions which have been proposed to explain the result from LSND.

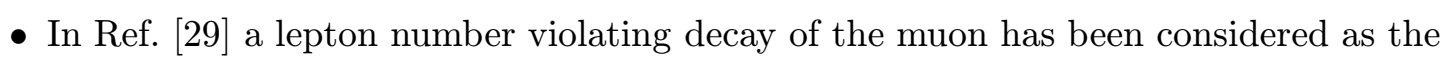
source for the LSND signal. In this model the $\bar{\nu}_{e}$ event excess in LSND stems from the decay $\mu^{+} \rightarrow e^{+}+\bar{\nu}_{e}+\bar{\nu}_{x}$, where $\bar{\nu}_{x}$ can be an anti-neutrino of any flavour. This scenario seems to be in disagreement with the non-observation of any $\bar{\nu}_{e}$ excess in KARMEN.

- Recently an explanation in terms of five neutrinos has been proposed [30ㅁㅁ. In such a $(3+2)$ scheme solar and atmospheric data are explained dominantly by active oscillations, similar to the $(3+1)$ case. The fit of SBL data is improved with respect to $(3+1)$ by involving two large mass splittings $\Delta m_{41}^{2} \sim 0.9 \mathrm{eV}^{2}$ and $\Delta m_{51}^{2} \sim 20 \mathrm{eV}^{2}$. In this case it seems to be very dificult to reconcile these large neutrino masses with

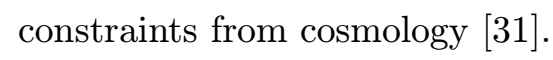

- In Ref. [i] $\overline{2} \overline{2}]$ it has been pointed out that involving a sterile neutrino and CPT violation simultaneously all the data can be explained. Once such drastical modifications of standard physics are accepted, one may adopt a $(3+1)$ like mass scheme, even with identical masses for neutrinos and anti-neutrinos, but allowing for different mixings. Since CDHS constrains only SBL muon neutrino disappearance (not anti-neutrinos), whereas Bugey restricts $\bar{\nu}_{e}$ mixing, a small violation of CPT would suffice to get around the SBL bounds and explain the LSND signal.

\section{Summary}

Performing a global analysis of current neutrino oscillation data we find that neither for four-neutrino schemes nor for CPT violation in a three-neutrino framework a satisfactory fit to the data is obtained.

- The strong rejection of non-active oscillation in the solar+KamLAND and atmospheric neutrino data rules out $(2+2)$ schemes, independent of whether LSND is confirmed or not. Using an improved goodness of fit method especially sensitive to the combination of data sets we find that $(2+2)$ schemes are ruled out at the $5.8 \sigma$ level.

- $(3+1)$ spectra are disfavoured by the disagreement of LSND with short-baseline disappearance data, leading to a marginal GOF of $5.6 \times 10^{-3}(3.2 \sigma)$. If LSND should be confirmed it would be very desirable to have more data on $\nu_{e}$ and/or $\nu_{\mu}$ SBL disappearance to decide about the status of $(3+1)$. In that case a positive signal is predicted right at the sensitivity edge of existing experiments.

- Also in the case of the CPT violating three-neutrino mass scheme no satisfactory fit can be obtained. This scheme is disfavoured by KamLAND, CHOOZ, Bugey and atmospheric anti-neutrino data at the $3.4 \sigma$ level. 
Alternative solutions, which have been proposed to reconcile the LSND result, seem to be in disagreement with some data, or require a very radical modification of standard physics. We conclude that currently no convincing explanation for the LSND result exists, and it remains a puzzle how to reconcile this evidence with the rest of the global data. Therefore, it is very important to settle this issue experimentally. A confirmation of the LSND signal by the MiniBooNE experiment [i] $3 \overline{3}$ i] would be very exciting and would require some new ideas.

\section{Acknowledgments}

I would like to thank M.C. Gonzalez-Garcia, M. Maltoni, M.A. Tórtola and J.W.F. Valle for collaboration on the work presented here. Furthermore, I thank the organisers for the very interesting workshop and for financial support. This work was supported by the "Sonderforschungsbereich 375-95 für Astro-Teilchenphysik" der Deutschen Forschungsgemeinschaft.

\section{References}

[1] S. Fukuda et al., Phys. Lett. B 539 (2002) 179; C. Yanagisawa, these proceedings; B.T. Cleveland et al., Astrophys. J. 496, 505 (1998); D.N. Abdurashitov et al., Phys. Rev. C60, 055801 (1999); astro-ph/0204245; W. Hampel et al., Phys. Lett. B447, 127 (1999); C. Cattadori, Nucl. Phys. B (Proc. Suppl.) 110 (2002) 311; Q. R. Ahmad et al., SNO Coll., Phys. Rev. Lett. 89, 011301 (2002); Phys. Rev. Lett. 89, 011302 (2002).

[2] Q. R. Ahmad et al., SNO Coll., N. Jelley, these proceedings.

[3] M. Shiozawa, Neutrino 2002, http://neutrino2002.ph.tum.de/; Y. Fukuda et al., Phys. Rev. Lett. 81 (1998) 1562; C. Yanagisawa, these proceedings.

[4] MACRO Coll., M. Ambrosio et al., Phys. Lett. B 434 (1998) 451; M. Giorgini, these proceedings.

[5] K. Eguchi et al., KamLAND Coll., Phys. Rev. Lett. 90 (2003) 021802; A. Piepke, these proceedings.

[6] M.H. Ahn et al., K2K Coll., Phys. Rev. Lett. 90, 041801 (2003), [hep-ex/0212007]; C. Yanagisawa, these proceedings.

[7] see e.g., M. Maltoni, T. Schwetz, M.A. Tórtola and J.W.F. Valle, hep-ph/0309130; M. Maltoni, these proceedings; G.L. Fogli, these proceedings.

[8] A. Aguilar et al., LSND Coll., Phys. Rev. D 64 (2001) 112007.

[9] J. T. Peltoniemi, D. Tommasini and J. W. F. Valle, Phys. Lett. B 298 (1993) 383;

J. T. Peltoniemi and J. W. F. Valle, Nucl. Phys. B 406, 409 (1993); D.O. Caldwell and R.N. Mohapatra, Phys. Rev. D 48 (1993) 3259.

[10] H. Murayama and T. Yanagida, Phys. Lett. B 520, 263 (2001); G. Barenboim, L. Borissov, J. Lykken and A. Y. Smirnov, JHEP 0210, 001 (2002).

[11] G. Barenboim, L. Borissov and J. Lykken, hep-ph/0212116.

[12] B. Armbruster et al., KARMEN Coll., Phys. Rev. D 65 (2002) 112001. 
[13] F. Dydak et al., CDHS Coll., Phys. Lett. B 134 (1984) 281.

[14] B. Achkar et al., Bugey Coll., Nucl. Phys. B 434 (1995) 503.

[15] M. Apollonio et al., CHOOZ Coll., Phys. Lett. B 466 (1999) 415; F. Boehm et al., Palo Verde Coll., Phys. Rev. D 64 (2001) 112001.

[16] M. Maltoni, T. Schwetz and J.W.F. Valle, Phys. Rev. D 65 (2002) 093004 [hep-ph/0112103].

[17] M. Maltoni, T. Schwetz, M.A. Tórtola and J.W.F. Valle, Nucl. Phys. B 643 (2002) 321 [hep-ph/0207157].

[18] M. Maltoni, T. Schwetz, M.A. Tórtola and J.W.F. Valle, Phys. Rev. D 67 (2003) 013011 [hep-ph/0207227].

[19] M.C. Gonzalez-Garcia, M. Maltoni and T. Schwetz, Phys. Rev. D 68, 053007 (2003) [hep-ph/0306226].

[20] M. Maltoni, T. Schwetz and J. W. Valle, Phys. Rev. D 67 (2003) 093003 [hep-ph/0212129]; T. Schwetz, Phys. Lett. B 577, 120 (2003) [hep-ph/0308003].

[21] M.C. Gonzalez-Garcia, M. Maltoni and C. Pena-Garay, Phys. Rev. D 64 (2001) 093001 [hep-ph/0105269].

[22] J.N. Bahcall, M.C. Gonzalez-Garcia and C. Pena-Garay, JHEP 0302 (2003) 009; G.L. Fogli, et al., Phys. Rev. D 67 (2003) 073002; G.L. Fogli, these proceedings; P.C. de Holanda and A.Y. Smirnov, JCAP 0302 (2003) 001; A.Y. Smirnov, these proceedings.

[23] M. Maltoni and T. Schwetz, Phys. Rev. D 68, 033020 (2003) [hep-ph/0304176].

[24] H. Pas, L. G. Song and T. J. Weiler, Phys. Rev. D 67 (2003) 073019 [hep-ph/0209373].

[25] S.M. Bilenky, C. Giunti and W. Grimus, Proc. of Neutrino '96, Helsinki; Eur. Phys. J. C 1 (1998) 247; N. Okada and O. Yasuda, Int. J. Mod. Phys. A 12 (1997) 3669; V. Barger, et at., Phys. Rev. D 58 (1998) 093016; S.M. Bilenky, C. Giunti, W. Grimus and T. Schwetz, Phys. Rev. D 60 (1999) 073007; O.L. Peres and A.Y. Smirnov, Nucl. Phys. B 599 (2001) 3; C. Giunti and M. Laveder, JHEP 0102 (2001) 001; W. Grimus and T. Schwetz, Eur. Phys. J. C 20 (2001) 1 [hep-ph/0102252].

[26] M. Maltoni, T. Schwetz and J. W. F. Valle, Phys. Lett. B 518, 252 (2001) [hep-ph/0107150].

[27] E.D. Church et al., Phys. Rev. D 66 (2002) 013001 [hep-ex/0203023].

[28] A. Strumia, Phys. Lett. B 539, 91 (2002), hep-ph/0201134.

[29] K. S. Babu and S. Pakvasa, hep-ph/0204236.

[30] M. Sorel, J. Conrad and M. Shaevitz, hep-ph/0305255.

[31] C.L. Bennett et al., astro-ph/0302207; D.N. Spergel et al., astro-ph/0302209; M. Tegmark et al., astro-ph/0310723; O. Elgaroy and O. Lahav, JCAP 0304 (2003) 004 [astro-ph/0303089]; S. Hannestad, JCAP 05 (2003) 004 [astro-ph/0303076]. P. Crotty, J. Lesgourgues and S. Pastor, Phys. Rev. D 67 (2003) 123005 [astro-ph/0302337]; J. Lesgourgues, these proceedings.

[32] V. Barger, D. Marfatia and K. Whisnant, Phys. Lett. B 576 (2003) 303 [hep-ph/0308299].

[33] E. Zimmerman, MiniBooNE Coll., hep-ex/0211039; I. Stancu, these proceedings. 